\title{
Management and outcome of moderate head trauma: our experience
}




\title{
Management and outcome of moderate head trauma: our experience
}

\author{
Mohammed T. Alshaimaa, Mohammed A. Sultan, Wael K. Zakaria, \\ Mohammed E. Elshehawi
}

Department of Neurosurgery, Mansoura University Hospital, Mansoura, EGYPT

\begin{abstract}
Objective: The aim of this study is to follow up patients with moderate head trauma who were admitted to Mansoura University Hospital in the period from 1 Dec. 2015 to 30 Jul. 2016 until discharge and determine the outcome of head trauma. Material and Methods: This prospective study were conducted on all patients with moderate head trauma admitted to Mansoura Emergency Hospital during the period from 1 Dec. 2015 to 30 Jul. 2016 with exclude Polytrauma, bleeding disorders, severe liver and kidney disease patients. Results: In this study, we correlated different risk factors with management and with outcome. Management may be surgical or conservative and outcome may be alive or dead. We have 60 patients with 17 cases $(28.3 \%)$ were treated surgically and 43 cases $(71.6 \%)$ were treated conservatively. According to outcome 36 cases $(60 \%)$ were alive and 24 cases $(40 \%)$ died, all cases managed in ICU. According to sex, 10 cases $(17 \%)$ were female and 50 cases were male $(83 \%)$ with statistically nonsignificant effect on outcome $(\mathrm{P}=0.7)$ and management $(\mathrm{P}=0.7)$. road traffic accidents is most common cause of injury with 33 cases (55\%), and Cause of injury had statistically significant effect on management $(\mathrm{P}=0.02)$ and statistically non-significant effect on outcome $(\mathrm{p}=0.4)$. GCS on admission had no statistically significant effect on management $(\mathrm{P}=0.8)$ and outcome $(\mathrm{P}=0.1)$ with mean of $10.1 \pm 1.2$ and $\mathrm{GCS}$ on discharge had no statistically significant effect on management $(\mathrm{P}=0.6)$. Conclusion: There were significant effect of age of patients, systemic diseases (such as DM, HTN, chronic kidney diseases, and chronic liver diseases), type of lesions (especially SDH, SAH), and serum electrolytes (especially serum Sodium) on outcome which determined by GCS at discharge, length of hospital stay, and the state of the patient at discharge.
\end{abstract}

Key words: Road Traffic accident, Glasgow coma scale

\section{Introduction}

In all of the world, Traumatic brain injury (TBI) considered as a critical public health and socio-economic problem. It is considered a major cause of death, in young adults, and result in lifelong disability in those who recovered $^{32,33}$. TBI looks like a silent epidemic, 
because unawareness of the society about the magnitude of this problem ${ }^{2840}$. In Egypt, TBI is a serious public health problem ${ }^{39}$.

Treating patients with TBI in USA cost about more than $9 \$$ billion per year ${ }^{49}$. TBI has two peaks of high incidence: first among young adult male between 15 to 24 years old, and other among elderly people of both sex older than 75 years ${ }^{3994041}$. Incidence in male is about $12-16 \%$ which double females incidence (8\%). Male have high incidence to be hospitalized and are nearly 3 times more to die from this injury ${ }^{3,10}$.

Most people who survived a head injury presented with a normal or mild deterioration of conscious level (Glasgow Coma Scale more than 12) with the majority of fatal outcomes happened in the moderate or severe head injury groups, which represent only $5 \%$ of cases. We defined Head injury as any trauma to the head other than any superficial injuries to the face ${ }^{39 \cdot 40}$. It can be classified by mechanism of injury to closed injury or penetrating injury, by morphology to fractures, focal intracranial injury, and diffuse injury, or by severity of injury to mild, moderate, and severe. Primary brain injury define as immediate brain damage happened upon time of impact. This includes different verities as cerebral contusions, diffuse axonal injuries, and acute subdural or epidural hematomas, subarachnoid hemorrhage and intracerebral hemorrhage. Secondary brain injury include other pathology as progressive cerebral oedema, ischemia, and increase size of cerebral contusions and the surrounding focal oedema, which lead to increase in intracranial pressure and can lead to cerebral herniation and death ${ }^{19340 \cdot 41}$.
Disabilities of TBI patients change according GCS: we have $47 \%$ moderate to severe disabilities at 12 months and a third do not return to work For patients with mild injuries (GCS 13-15),. For patients with moderate brain injuries (GCS 9-12), moderate to severe disabilities are $45 \%$, and while $48 \%$ of severely injured patients have moderate to severe disabilities, only $14 \%$ have a good outcome at one year after trauma ${ }^{49,55}$.

\section{Pathophysiology of TBI:}

We divided traumatic brain injury into the primary neuronal injury which followed by secondary injury. The primary injury define as the initial injury of neuron that occurs immediately at time of impact. ${ }^{4,22}$. While secondary injury occurred minutes, hours or days after injury impact and lead to worsen the primary lesions ${ }^{57,59}$.

Secondary injury considered as cellular and molecular processes which started by the primary injury and aggravated by the cerebral damage as result of hypotensive or hypoxic events, hypoglycemia, and elevated intracranial pressure cause cerebral ischemia. Andrriesson ${ }^{3 / 4}$. Glutamate excitotoxicity, neuronal depolarization, disturbance of ionic homeostasis, nitric oxide and oxygen free radicals, lipid peroxidation, disruption of blood-brain barrier, cerebral edema and ischemia, mitochondrial dysfunction, axonal disruption and necrotic cell death considered as mechanisms of secondary injury ${ }^{38} 45$.

TBI start a close circle of neurotoxic phenomena which aggravate each other and end finally in cell death either by apoptosis or cell necrosis ${ }^{59}$.

\section{Diagnosis of TBI:}

Assessing the GCS and size of pupils and 
reaction to light considered as initial neurologic examination. Prove alcohol intoxication lowers initial GCS in TBI patients 18,24

Computed tomography (CT) of the head is used for both confirming TBI and follow up patients over time after impact. CT scan can be used to know the type and severity of the injury; with upper hand for detecting intracranial hematoms ${ }^{32} \cdot 33.50 \%$ of moderate TBI patients have abnormal finding in CT $\operatorname{scan}^{19}$.

\section{Treatment:}

Treatment divided into: pre-hospital, casualty department, and other, which includes both surgical treatment and intensive care unit treatment ${ }^{8 \cdot 9}$.

\section{Prognosis and Outcome:}

Mortality in moderate TBI is $15 \%$, and 75 $\%$ of these deaths happen in sever injured patients ${ }^{1922}$. More than $50 \%$ of the survivors after moderate TBI suffer cognitive problem and whose recover without significant disability only $20 \%{ }^{21}$.

Death incidence 3.5 years after injury in teenagers and adults affected by moderate or severe TBI who were discharged from hospital after treatment was more than twice compared to persons in the general population of similar age, sex, and race ${ }^{3,19}$.

There are many predictors factors of outcome after TBI include GCS after resuscitation, age, pupillary reactivity, CT findings, and the presence of major associated extra-cranial injury ${ }^{45}, 57$.

\section{Material and Methods}

This prospective study, after approval by the local ethical committee of anesthesia \& surgical intensive care department Mansoura university hospital were conducted on all patients with head trauma admitted to Mansoura Emergency Hospital during the period from 1 Dec. 2015 to 30 Jul. 2016.

\section{Inclusion criteria:}

Patients with head trauma only, different age group (pediatrics from 0 to 15 years, adults from 15 to 65 year, geriatrics more than 65 year)., patients with moderate head injury GCS (9-12), patients with intracranial hemorrhage, patients with skull fracture depressed or linear, patients with cerebral contusions, patients treated surgically and treated conservative, drug abuse patients and alcohol drinkers, diabetic patients smokers, controlled hypertensive patient, patient with mild liver disease and mild renal disease controlled with treatment.

\section{Exclusion criteria:}

Polytrauma patients (abdominal injury, chest injury, fracture spine, bone fractures), patients with bleeding disorders, patients on anticoagulant therapy, severe liver disease patients, severe kidney disease patients, mild head injury GCS (13-15) and severe head injury GCS (8 or less).

\section{Results}

In this study, we correlated different risk factors with management and outcome. Management may be surgical or conservative and outcome may be alive or dead. We found that total number of cases treated surgically was 17 cases (28.3\%) and 43 cases (71.6\%) were treated conservatively. According to outcome 36 cases $(60 \%)$ were alive and 24 cases $(40 \%)$ 
died, all cases managed in ICU, figures $(1,2)$.

In this study, total number of cases was divided into 3 groups according to age: 1) Children: 16 cases $(26.7 \%)$ with age ranging from 1 month to 16 years. 2) Adult: 38 cases (63.3\%) with age ranging from 17 years to 59 years. 3) Elderly: 6 cases (10\%) with age $\geq 60$ years, table (1) and figure (3).

According to sex, 10 cases (17\%) were female and 50 cases were male (83\%), according to $\mathrm{P}$ value sex had statistically nonsignificant effect on outcome $(\mathrm{P}=0.7)$ and management ( $\mathrm{P}=0.7)$, table (2) and figure (4).

According to the cause of injury, falls was 19 cases $(31.6 \%)$, road traffic accidents (RTA) 33 cases $(55 \%)$, struggle 4 cases $(6.6 \%)$ and direct head trauma (DHT) 4 cases (6.6\%). Cause of injury had statistically significant effect on management $(\mathrm{P}=0.02)$ and statistically non-significant effect on outcome $(\mathrm{p}=0.4)$, as shown in table $(3,4)$ and figure (5).

In this study, patients who had DM 10 cases, 9 cases had HTN, only 1 case had history of drug abuse and 6 cases had other medical disorder e.g. CLD, CKD, epilepsy, cardiac diseases. These cases might have only one disease or might have more than one systemic diseases, as shown in table (5).

GCS on admission had no statistically significant effect on management ( $\mathrm{P}$ value $=0.8)$ and outcome $(\mathrm{P}=0.1)$ with mean of $10.1 \pm 1.2$ and GCS on discharge had no statistically significant effect on management $(\mathrm{P}=0.6)$, as shown in table (6).

In our study, we depended on CT diagnosis to illustrate type of TBI and its relation to management and outcome. Patients who had EDH were 13 cases, 20 cases had SDH, 8 cases had SAH, 18 cases had contusions, 2 cases had
ICH, 4 cases had DAI, 12 cases had brain edema, 19 cases had fissure fracture, 2 cases had depressed fracture, 1 case had fracture base of skull, 5cases had pneumocephalus, tables $(7,8,9)$ and figure $(6)$.

According to measured data we observed that blood pressure had statistically significant effect on outcome ( $\mathrm{P}$ value $=0.006$ for systolic $\mathrm{BP}$ and $\mathrm{P}$ value $=0.002$ for diastolic $\mathrm{BP}$ ) and non-significant effect on management ( $\mathrm{P}$ value $=0.2$ for systolic $B P \& P=0.1$ for diastolic BP). Arterial Blood Gases (ABG) had statistically significant effect $(P=0.04)$ on management and no statistically significance on outcome $(\mathrm{P}=0.7)$. Serum sodium $(\mathrm{Na}$.) value had statistically significant effect in outcome $(\mathrm{P}=0.004)$ while it had no statistically significant effect on management $(\mathrm{P}=0.5)$. Serum potassium (K.) had neither statistically significant effect on management $(\mathrm{P}=0.3)$ nor on outcome $(\mathrm{P}=0.2)$. INR in most cases was within normal range with mean $1.2 \pm 0.2$ which had neither statistically significant effect on management $(\mathrm{P}=0.9)$ nor on outcome $(\mathrm{P}=0.4)$. Liver enzymes (ALT) had no statistically significant effect on management $(\mathrm{P}=0.08)$ while it had statistically significant effect on outcome ( $\mathrm{P}=0.03)$. All cases had leukocytosis (normal WBCs 4000-11000) which had neither statistically significant effect on outcome ( $\mathrm{P}=0.9)$ nor management $(\mathrm{p}=0.07)$. Hemoglobin (Hg.) had neither statistically significant effect on management $(\mathrm{P}=0.7)$ nor outcome $(\mathrm{P}=0.1)$. Platelets had neither statistically significant effect on management $(\mathrm{P}=0.6)$ nor outcome $(\mathrm{P}=0.3)$. Serum creatinine had statistically non-significant effect on management $(\mathrm{P}=0.6)$ while it had statistically significant effect on outcome 
$(\mathrm{P} \leq 0.001)$. Random Blood Sugar (RBS) had no statistically significant effect on management $(\mathrm{P}=0.4)$ while it had statistically significant effect on outcome $(\mathrm{P} \leq 0.001)$, as shown in table (10).

According to the duration of hospital stay, survived patients had longer duration of hospital stay than dead patients with mean duration of hospital stay for survived 8(4-45) and for dead 7(1-27), which had statistically non-significant effect on outcome $(\mathrm{P}=1)$. Patients treated surgically had longer duration of hospital stay than treated conservatively with mean of duration of hospital stay for surgical treatment $8 \quad(2-45)$ and for conservative treatment $7.5(1-30)$ day which had no statistically significant effect on management $(\mathrm{P}=1)$, as shown in figures $(7,8)$.

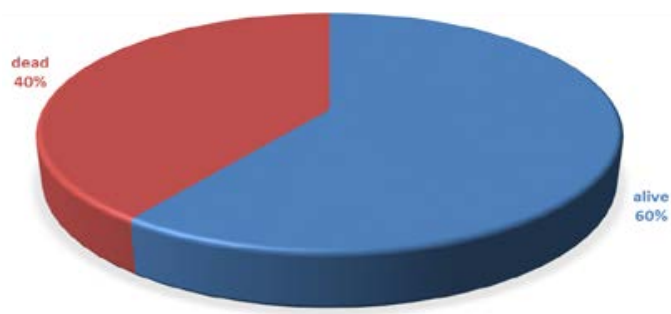

Figure 1 - illustrates percentage of dead to alive

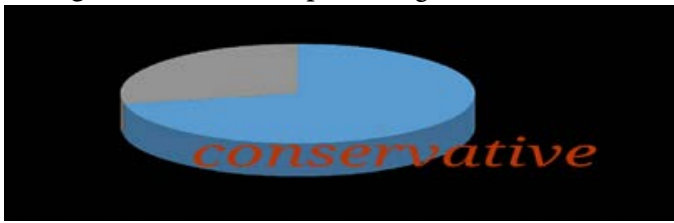

Figure 2 illustrates percentage of surgical to conservative treatment

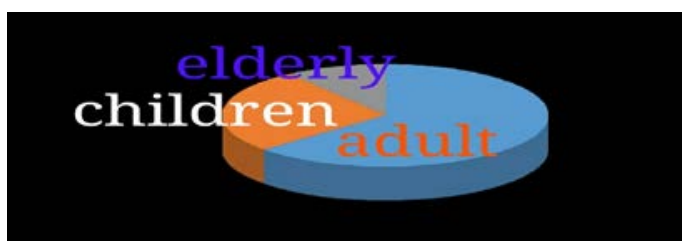

Figure 3 illustrates age groups

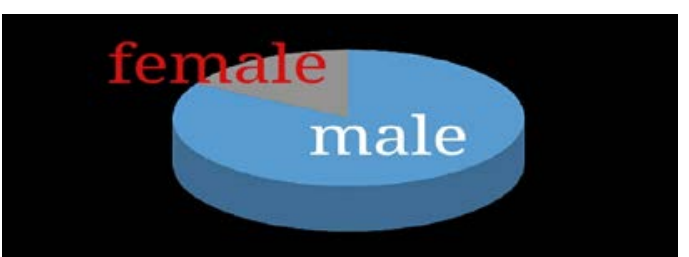

Figure 4 illustrates percentage of male to female

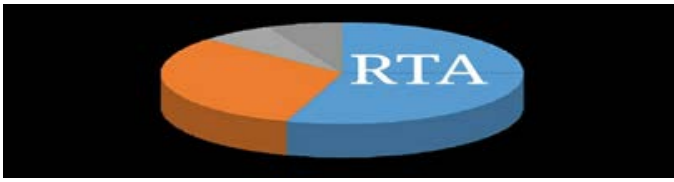

Figure 5 illustrates cause of injury

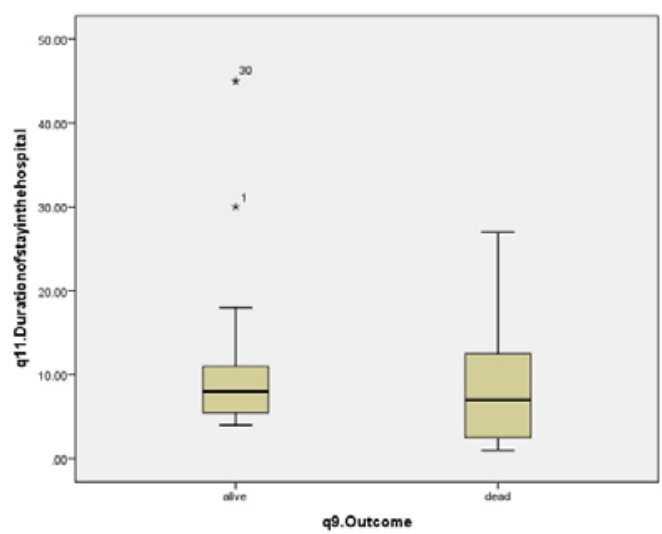

Figure 6 illustrates relation between hospital stay and outcome

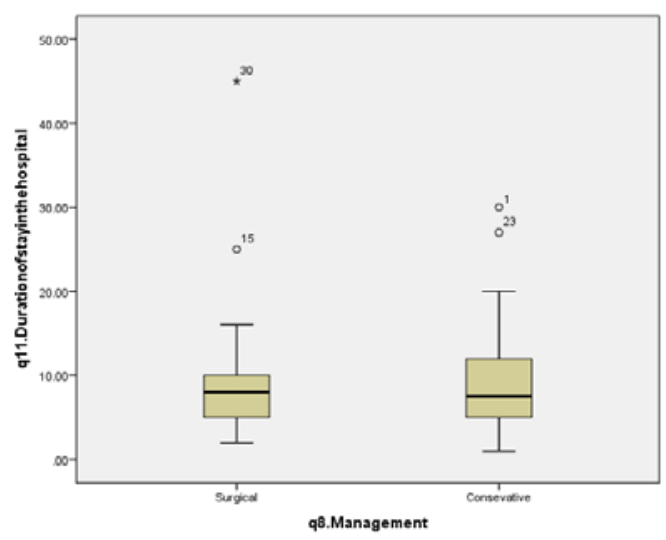

Figure 7 illustrates relation of hospital stay to management 
TABLE I

Correlates age groups to management and outcome

\begin{tabular}{|l|c|c|c|c|c|c|c|c|}
\hline & \multicolumn{3}{|c|}{ Management } & \multicolumn{3}{c|}{ Outcome } \\
\hline & Surgical & Conservative & Significance test & Alive & \multicolumn{2}{c|}{ Dead } & \multicolumn{2}{c|}{ Significance test } \\
& $\mathrm{N}=17$ & $\mathrm{~N}=43$ & & $\mathrm{~N}=36$ & $\mathrm{~N}=24$ \\
& $\mathrm{No}(\%)$ & $\mathrm{No}(\%)$ & & $\mathrm{No}(\%)$ & $\mathrm{No}(\%)$ & & $\mathrm{MCT}$ \\
\hline Child & $3(17.6)$ & $13(30.2)$ & $\mathrm{P}=0.5$ & $\mathrm{MCT}$ & $15(41.7)$ & $1(4.2)$ & $\mathrm{P}=0.003^{*}$ & $\mathrm{P}=0.001^{*}$ \\
\hline Adult & $13(76.5)$ & $25(58.1)$ & $\mathrm{P}=0.3$ & & $20(55.6)$ & $18(75)$ & $\mathrm{P}=0.1$ & \\
\hline Elderly & $1(5.9)$ & $5(11.6)$ & $\mathrm{P}=0.8$ & & $1(2.8)$ & $5(20.8)$ & $\mathrm{P}=0.07$ & \\
\hline
\end{tabular}

TABLE II

Correlates sex to management and outcome

\begin{tabular}{|c|c|c|c|c|c|c|}
\hline & \multicolumn{3}{|c|}{ Management } & \multicolumn{3}{|c|}{ Outcome } \\
\hline & $\begin{array}{l}\text { Surgical } \\
\mathrm{N}=17 \\
\text { No }(\%)\end{array}$ & $\begin{array}{l}\text { Conservative } \\
\qquad \begin{array}{l}\mathrm{N}=43 \\
\text { No }(\%)\end{array}\end{array}$ & Significance test & $\begin{array}{c}\text { Alive } \\
\mathrm{N}=36 \\
\text { No }(\%)\end{array}$ & $\begin{array}{c}\text { Dead } \\
\mathrm{N}=24 \\
\text { No }(\%)\end{array}$ & Significance test \\
\hline $\begin{array}{l}\text { Age:median } \\
(\min -\max )\end{array}$ & $25(2-74)$ & $20(0.5-74)$ & $\mathrm{Z}=0.3, \mathrm{P}=0.7$ & $18.5(0.5-71)$ & $49.5(5-74)$ & $\mathrm{Z}=4.1, \mathrm{P} \leq 0.001^{\star}$ \\
\hline female & $2(11.8)$ & $8(18.6)$ & $\begin{array}{c}\text { FET } \\
\mathrm{P}=0.7\end{array}$ & $7(19.4)$ & $3(12.5)$ & $\begin{array}{c}\text { FET } \\
\mathrm{P}=0.7\end{array}$ \\
\hline male & $15(88.2)$ & $35(81.4)$ & & $29(80.6)$ & $21(87.5)$ & \\
\hline
\end{tabular}

Z of Mann-Whitney test SD: standard deviation

FET: Fisher's Exact Test MCT: Monte Carlo Test

P: Probability $\quad P$ value is significant if $\leq 0.05$, highly significant if $\leq 0.0001$ 


\section{TABLE III}

\section{Correlates cause of injury to management and outcome}

\begin{tabular}{|c|c|c|c|c|c|c|c|c|}
\hline & \multicolumn{4}{|c|}{ Management } & \multicolumn{4}{|c|}{ Outcome } \\
\hline & Surgical & Conservative & \multicolumn{2}{|c|}{ Significance test } & Alive & Dead & \multicolumn{2}{|c|}{ Significance test } \\
\hline & $\mathrm{N}=17$ & $\mathrm{~N}=43$ & & & $\mathrm{~N}=36$ & $\mathrm{~N}=24$ & & \\
\hline & No (\%) & No $(\%)$ & & & No (\%) & No (\%) & & \\
\hline falls & $2(11.8)$ & $17(39.5)$ & $\mathrm{P}=0.08$ & MCT & $14(38.9)$ & $5(20.8)$ & $\mathrm{P}=0.2$ & MCT \\
\hline RTA & $12(70.6)$ & $21(48.8)$ & $\mathrm{P}=0.2$ & & $17(47.2)$ & $16(66.7)$ & $\mathrm{P}=0.2$ & \\
\hline struggle & $3(17.6)$ & $1(2.3)$ & $\mathrm{P}=0.1$ & & $2(5.6)$ & $2(8.3)$ & $\mathrm{P}=1$ & \\
\hline DHT & $0(0)$ & $4(9.3)$ & $\mathrm{P}=0.5$ & & $3(8.3)$ & $1(4.2)$ & $\mathrm{P}=0.9$ & \\
\hline
\end{tabular}

${ }^{\star}$ There is significant correlation between cause of injury and management $(\mathrm{P}=0.02)$

\section{TABLE IV}

Correlates cause of injury to age groups

\begin{tabular}{|c|c|c|c|c|c|}
\hline & \multicolumn{4}{|c|}{ Cause of injury } & \multirow{2}{*}{ Significance test } \\
\hline & $\begin{array}{l}\text { Falls } \\
\mathrm{n}=19\end{array}$ & $\begin{array}{l}\text { RTA } \\
n=33\end{array}$ & $\begin{array}{l}\text { Struggle } \\
\qquad n=4\end{array}$ & $\begin{array}{l}\text { DHT } \\
n=4\end{array}$ & \\
\hline \multicolumn{6}{|c|}{ Age group } \\
\hline Child & $10(52.6)$ & $4(12.1)$ & $0(0)$ & $2(50)$ & $\begin{array}{c}\text { MCT } \\
\mathrm{P}=0.001^{\star}\end{array}$ \\
\hline Adult & $4(21.1)$ & $28(84.8)$ & $4(100)$ & $2(50)$ & \\
\hline Elderly & $5(26.3)$ & $1(3)$ & $0(0)$ & $0(0)$ & \\
\hline
\end{tabular}

${ }^{\star}$ There is statistically significant correlation between age of patient and the cause of injury $(\mathrm{P}=0.001)$ 


\section{TABLE V}

Correlates systemic diseases to management and outcome

\begin{tabular}{|c|c|c|c|c|c|c|}
\hline & \multicolumn{3}{|c|}{ Management } & \multicolumn{3}{|c|}{ outcome } \\
\hline & $\begin{array}{c}\text { Surgical } \\
\mathrm{N}=17 \\
\text { No }(\%)\end{array}$ & $\begin{array}{c}\text { Conservative } \\
\mathrm{N}=43 \\
\mathrm{No}(\%)\end{array}$ & Significance test & $\begin{array}{c}\text { Alive } \\
\mathrm{N}=36 \\
\text { No (\%) }\end{array}$ & $\begin{array}{c}\text { Dead } \\
\mathrm{N}=24 \\
\text { No }(\%)\end{array}$ & Significance test \\
\hline $\mathrm{DM}$ & $3(17.6)$ & $7(16.3)$ & $\begin{array}{l}\text { FET } \\
\mathrm{P}=1\end{array}$ & $1(2.8)$ & $9(37.5)$ & $\begin{array}{c}\text { FET } \\
\mathrm{P}=0.001^{*}\end{array}$ \\
\hline Hypertension & $1(5.9)$ & $8(18.6)$ & $\begin{array}{c}\text { FET } \\
\mathrm{P}=0.4\end{array}$ & $1(2.8)$ & $8(33.3)$ & $\begin{array}{c}\text { FET } \\
\mathrm{P}=0.002^{*}\end{array}$ \\
\hline Drug abuse & $1(5.9)$ & $0(0)$ & $\begin{array}{c}\text { FET } \\
\mathrm{P}=0.3\end{array}$ & $1(2.8)$ & $0(0)$ & $\begin{array}{l}\text { FET } \\
\mathrm{P}=1\end{array}$ \\
\hline $\begin{array}{c}\text { Other systemic } \\
\text { diseases..... }\end{array}$ & $1(5.9)$ & $5(11.6)$ & $\begin{array}{c}\text { FET } \\
\mathrm{P}=0.7\end{array}$ & $2(5.6)$ & $4(16.7)$ & $\begin{array}{c}\text { FET } \\
\mathrm{P}=0.2\end{array}$ \\
\hline
\end{tabular}

${ }^{\star}$ There is significant correlation between DM, HTN and outcome $(\mathrm{P}=0.001),(\mathrm{P}=0.002)$ respectively

TABLE VI

Correlates GCS to management and outcome

\begin{tabular}{|c|c|c|c|c|c|c|c|c|}
\hline & \multicolumn{4}{|c|}{ Management } & \multicolumn{4}{|c|}{ Outcome } \\
\hline & $\begin{array}{c}\text { Surgical } \\
\mathrm{N}=17 \\
\text { No }(\%)\end{array}$ & \begin{tabular}{|c|} 
Conservative \\
$\mathrm{N}=43$ \\
$\mathrm{No}(\%)$
\end{tabular} & \multicolumn{2}{|c|}{ Significance test } & $\begin{array}{c}\text { Alive } \\
\mathrm{N}=36 \\
\text { No (\%) }\end{array}$ & $\begin{array}{c}\text { Dead } \\
\mathrm{N}=24 \\
\text { No }(\%)\end{array}$ & \multicolumn{2}{|c|}{ Significance test } \\
\hline Initial GCS & $10.1 \pm 1.2$ & $10.3 \pm 1.2$ & \multicolumn{2}{|c|}{$P=0.6$} & $10.2778 \pm 1.1$ & $10.1 \pm 1.3$ & \multicolumn{2}{|c|}{$\mathrm{P}=0.6$} \\
\hline 9.00 & $8(47.1)$ & $14(32.6)$ & $\mathrm{P}=0.9$ & $\begin{array}{l}\text { MCT } \\
\mathrm{P}=0.8\end{array}$ & $10(27.8)$ & $12(50)$ & $\mathrm{P}=0.08$ & $\begin{array}{l}\text { MCT } \\
\mathrm{P}=0.1\end{array}$ \\
\hline 10.00 & $3(17.6)$ & $12(27.9)$ & $\mathrm{P}=0.6$ & & $12(33.3)$ & $3(12.5)$ & $\mathrm{P}=0.1$ & \\
\hline 11.00 & $3(17.6)$ & $8(18.6)$ & $\mathrm{P}=1$ & & $8(22.2)$ & $3(12.5)$ & $P=0.5$ & \\
\hline 12.00 & $3(17.6)$ & $9(20.9)$ & $\mathrm{P}=1$ & & $6(16.7)$ & $6(25)$ & $\mathrm{P}=0.4$ & \\
\hline \multicolumn{9}{|c|}{ GCS on discharge } \\
\hline 14.00 & $2(4.3)$ & $7(28.6)$ & \multicolumn{2}{|c|}{$\begin{array}{c}\text { FET } \\
\mathrm{P}=0.6\end{array}$} & & & & \\
\hline 15.00 & $7(35.7)$ & $20(71.4)$ & & & & & & \\
\hline
\end{tabular}




\section{TABLE VII}

Correlates type of lesion to management and outcome according to CT on admission

\begin{tabular}{|c|c|c|c|c|c|c|}
\hline & \multicolumn{3}{|c|}{ Management } & \multicolumn{3}{|c|}{ Outcome } \\
\hline & $\begin{array}{c}\text { Surgical } \\
\mathrm{N}=17 \\
\text { No }(\%)\end{array}$ & $\begin{array}{c}\text { Conservative } \\
\begin{array}{c}\mathrm{N}=43 \\
\mathrm{No}(\%)\end{array}\end{array}$ & Significance test & $\begin{array}{c}\text { Alive } \\
\mathrm{N}=36 \\
\text { No }(\%)\end{array}$ & $\begin{array}{c}\text { Dead } \\
\mathrm{N}=24 \\
\text { No }(\%)\end{array}$ & Significance test \\
\hline $\begin{array}{cc}\text { EDH: } & \\
& \text { Only } \\
\text { Other lesion }\end{array}$ & $\begin{array}{l}4(44.4) \\
5(55.6) \\
\end{array}$ & $\begin{array}{l}1(33.3) \\
3(66.7) \\
\end{array}$ & $\begin{array}{l}\text { FET } \\
P=1\end{array}$ & $\begin{array}{l}5(50) \\
5(50) \\
\end{array}$ & $\begin{array}{c}0(0) \\
3(100) \\
\end{array}$ & $\begin{array}{c}\text { FET } \\
\mathrm{P}=0.2\end{array}$ \\
\hline \begin{tabular}{|l} 
SDH: \\
$\quad$ Only \\
\\
And other lesion
\end{tabular} & $\begin{array}{l}3(33.3) \\
6(66.7)\end{array}$ & $\begin{array}{l}5(45.5) \\
6(54.5) \\
\end{array}$ & $\begin{array}{c}\text { FET, } \\
\mathrm{P}=0.7\end{array}$ & $\begin{array}{l}4(57.1) \\
3(42.9)\end{array}$ & $\begin{array}{l}4(30.8) \\
9(69.2)\end{array}$ & $\begin{array}{c}\text { FET } \\
\mathrm{P}=0.4\end{array}$ \\
\hline \begin{tabular}{|l} 
SAH: \\
$\quad$ Only \\
\\
And other lesion
\end{tabular} & $\begin{array}{l}0(0) \\
0(0)\end{array}$ & $\begin{array}{l}2(12.5) \\
6(87.5)\end{array}$ & $\begin{array}{l}\text { FET } \\
P=1\end{array}$ & $\begin{array}{c}0(0) \\
1(100)\end{array}$ & $\begin{array}{l}2(28.6) \\
5(71.4)\end{array}$ & $\begin{array}{l}\text { FET } \\
P=1\end{array}$ \\
\hline $\begin{array}{c}\text { Contusion: } \\
\text { Only } \\
\text { And other lesion }\end{array}$ & $\begin{array}{c}0 \\
2(100)\end{array}$ & $\begin{array}{c}2(100) \\
14(87.5)\end{array}$ & $\begin{array}{l}\text { FET } \\
P=1\end{array}$ & $\begin{array}{l}2(25) \\
6(75)\end{array}$ & $\begin{array}{c}0(0) \\
10(100)\end{array}$ & $\begin{array}{c}\text { FET } \\
\mathrm{P}=0.2\end{array}$ \\
\hline $\begin{array}{ll}\mathrm{ICH}: & \\
& \text { Only } \\
& \text { And other lesion }\end{array}$ & $\begin{array}{l}0(0) \\
0(0)\end{array}$ & $\begin{array}{l}1(50) \\
1(50)\end{array}$ & $\begin{array}{l}\text { FET } \\
P=1\end{array}$ & $\begin{array}{c}0(0) \\
1(100)\end{array}$ & $\begin{array}{c}1(100) \\
0(0)\end{array}$ & $\begin{array}{l}\text { FET } \\
P=1\end{array}$ \\
\hline $\begin{array}{c}\text { Fissure fracture: } \\
\text { Only } \\
\text { And other lesion }\end{array}$ & $\begin{array}{c}0 \\
7(100)\end{array}$ & $\begin{array}{c}2(16.7) \\
10(83.3)\end{array}$ & $\begin{array}{c}\text { FET } \\
\mathrm{P}=0.5\end{array}$ & $\begin{array}{l}2(18.2) \\
9(81.8)\end{array}$ & $\begin{array}{c}0(0) \\
8(100)\end{array}$ & $\begin{array}{c}\text { FET } \\
\mathrm{P}=0.5\end{array}$ \\
\hline $\begin{array}{c}\text { depressed Fracture: } \\
\text { Only } \\
\text { And other lesion } \\
\end{array}$ & $\begin{array}{c}0 \\
1(100) \\
\end{array}$ & $\begin{array}{c}0(0) \\
1(100)\end{array}$ & $\begin{array}{l}\text { FET } \\
\mathrm{P}=1\end{array}$ & $\begin{array}{c}0(0) \\
1(100)\end{array}$ & $\begin{array}{c}0(0) \\
1(100)\end{array}$ & $\begin{array}{l}\text { FET } \\
\mathrm{P}=1\end{array}$ \\
\hline $\begin{array}{c}\text { Fracture base skull: } \\
\text { Only } \\
\text { And other lesion }\end{array}$ & $\begin{array}{l}0 \\
0\end{array}$ & $\begin{array}{c}0(0) \\
1(100)\end{array}$ & $\begin{array}{l}\text { FET } \\
\mathrm{P}=1\end{array}$ & $\begin{array}{c}0(0) \\
1(100)\end{array}$ & $\begin{array}{l}0(0) \\
0(0)\end{array}$ & $\begin{array}{l}\text { FET } \\
\mathrm{P}=1\end{array}$ \\
\hline $\begin{array}{c}\text { Brain edema: } \\
\text { Only } \\
\text { And other lesion }\end{array}$ & $\begin{array}{l}0 \\
0\end{array}$ & $\begin{array}{l}4(33.3) \\
8(66.7)\end{array}$ & $\begin{array}{l}\text { FET } \\
P=1\end{array}$ & $\begin{array}{l}3(33.3) \\
6(66.7)\end{array}$ & $\begin{array}{l}1(33.3) \\
2(66.7)\end{array}$ & $\begin{array}{c}\text { FET } \\
\mathrm{P}=0.4\end{array}$ \\
\hline $\begin{array}{l}\text { DAI: } \\
\quad \text { Only DAI } \\
\text { DAI and other lesion }\end{array}$ & $\begin{array}{r}0(0) \\
0(0)\end{array}$ & $\begin{array}{c}4(100) \\
0\end{array}$ & $\begin{array}{l}\text { FET } \\
P=1\end{array}$ & $\begin{array}{c}4(100) \\
0(0)\end{array}$ & $\begin{array}{l}0(0) \\
0(0)\end{array}$ & $\begin{array}{c}\text { FET } \\
\mathrm{P}=0.1\end{array}$ \\
\hline $\begin{array}{c}\text { Pneumocephalus: } \\
\text { Only } \\
\text { And other lesion }\end{array}$ & $\begin{array}{c}0(0) \\
2(100)\end{array}$ & $\begin{array}{c}0(0) \\
3(100)\end{array}$ & $\begin{array}{l}\text { FET } \\
P=1\end{array}$ & $\begin{array}{c}0(0) \\
5(100)\end{array}$ & $\begin{array}{l}0(0) \\
0(0)\end{array}$ & $\begin{array}{c}\text { FET } \\
\mathrm{P}=0.07\end{array}$ \\
\hline
\end{tabular}


TABLE VIII

Correlates type of lesion to outcome

\begin{tabular}{|c|c|c|c|}
\hline & \multicolumn{2}{|c|}{ Outcome } & \multirow[t]{2}{*}{ Significance test } \\
\hline & $\begin{array}{l}\text { Alive } \\
\mathrm{n}=36\end{array}$ & $\begin{array}{l}\text { Dead } \\
\mathrm{n}=24\end{array}$ & \\
\hline & No (\%) & No $(\%)$ & \\
\hline EDH & $10(27.8)$ & $3(12.5)$ & $\begin{array}{l}\aleph^{2}=1.9 \\
\mathrm{P}=0.2\end{array}$ \\
\hline $\mathrm{SDH}$ & $7(19.4)$ & $13(54.2)$ & $\begin{array}{c}\aleph^{2}=7.8 \\
P=0.005^{*}\end{array}$ \\
\hline SAH & $1(2.8)$ & $7(29.2)$ & $\begin{array}{c}\text { FET } \\
\mathrm{P}=0.005^{*}\end{array}$ \\
\hline Contusion & $8(22.2)$ & $10(41.7)$ & $\begin{array}{c}\aleph^{2}=2.6 \\
P=0.1\end{array}$ \\
\hline $\mathrm{ICH}$ & $1(2.8)$ & $1(4.2)$ & $\begin{array}{l}\text { FET } \\
\mathrm{P}=1\end{array}$ \\
\hline Fissure fracture & $11(30.6)$ & $8(33.3)$ & $\begin{array}{c}\aleph^{2}=0.05 \\
\mathrm{P}=0.8\end{array}$ \\
\hline Fracture depressed & $1(2.8)$ & $1(4.2)$ & $\begin{array}{l}\text { FET } \\
\mathrm{P}=1\end{array}$ \\
\hline Fracture base of the skull & $1(2.8)$ & $0(0)$ & $\begin{array}{l}\text { FET } \\
\mathrm{P}=1\end{array}$ \\
\hline Brain edema & $9(25)$ & $3(12.5)$ & $\begin{array}{c}\text { FET } \\
\mathrm{P}=0.3\end{array}$ \\
\hline DAI & $4(11.1)$ & $0(0)$ & $\begin{array}{c}\text { FET } \\
P=0.1\end{array}$ \\
\hline Pneumocephalus & $5(13.9)$ & $0(0)$ & $\begin{array}{c}\text { FET } \\
\mathrm{P}=0.08\end{array}$ \\
\hline
\end{tabular}

${ }^{\star}$ There is significant correlation between $\mathrm{SDH}, \mathrm{SAH}$ and outcome $(\mathrm{P}=0.005),(\mathrm{P}=0.005)$ respectively. 
TABLE IX

Correlates age with type of lesion

\begin{tabular}{|c|c|c|c|c|}
\hline & $\begin{array}{l}\text { Child } \\
\text { n=16 } \\
\text { No (\%) }\end{array}$ & $\begin{array}{l}\text { Adult } \\
\mathrm{n}=38 \\
\text { No (\%) }\end{array}$ & $\begin{array}{c}\text { Elderly } \\
n=6 \\
\text { No (\%) }\end{array}$ & Significance test \\
\hline $\mathrm{EDH}$ & $3(18.8)$ & $9(25)$ & $1(12.5)$ & $\begin{array}{c}\text { MCT } \\
\mathrm{P}=0.7\end{array}$ \\
\hline SDH & $1(6.3)$ & $15(41.7)^{\star}$ & $4(50)$ & $\begin{array}{c}\text { MCT } \\
\mathrm{P}=0.03^{\star}\end{array}$ \\
\hline $\mathrm{SAH}$ & $1(6.3)$ & $5(13.9)$ & $2(25)$ & $\begin{array}{c}\text { MCT } \\
\mathrm{P}=0.5\end{array}$ \\
\hline Contusion & $5(31.3)$ & $10(27.8)$ & $3(37.5)$ & $\begin{array}{c}\text { MCT } \\
\mathrm{P}=0.9\end{array}$ \\
\hline $\mathrm{ICH}$ & $0(0)$ & $1(2.8)$ & $1(12.5)$ & $\begin{array}{c}\text { MCT } \\
\mathrm{P}=0.3 \\
\end{array}$ \\
\hline Fracture: fissure & $9(56.3)$ & $10(27.8)^{*}$ & $0(0)$ & $\begin{array}{c}\text { MCT } \\
\mathrm{P}=0.02^{\star}\end{array}$ \\
\hline Fracture: depressed & $0(0)$ & $2(5.6)$ & $0(0)$ & $\begin{array}{l}\text { MCT } \\
\mathrm{P}=0.7\end{array}$ \\
\hline Fracture base skull & $1(6.3)$ & $0(0)$ & $0(0)$ & $\begin{array}{l}\text { MCT } \\
\mathrm{P}=0.4 \\
\end{array}$ \\
\hline Brain edema & $5(31.3)$ & $7(19.4)$ & $0(0)$ & $\begin{array}{l}\text { MCT } \\
\mathrm{P}=0.2\end{array}$ \\
\hline DAI & $1(6.3)$ & $3(8.3)$ & $0(0)$ & $\begin{array}{l}\text { MCT } \\
\mathrm{P}=0.8\end{array}$ \\
\hline Pneumocephalus & $2(12.5)$ & $3(8.3)$ & $0(0)$ & $\begin{array}{l}\text { MCT } \\
\mathrm{P}=0.7\end{array}$ \\
\hline
\end{tabular}

${ }^{\star}$ There is significant correlation between age of patients and type of lesion $\mathrm{SDH}$, fissure fracture $(\mathrm{P}=0.03)$, $(\mathrm{P}=0.02)$ respectively. 


\section{TABLE X}

Correlates lab. Data to management and outcome

\begin{tabular}{|c|c|c|c|c|c|c|}
\hline \multirow[t]{2}{*}{ Parameter } & \multicolumn{3}{|c|}{ Management } & \multicolumn{3}{|c|}{ Outcome } \\
\hline & $\begin{array}{l}\text { Surgical } \quad \mathrm{n}= \\
17 \\
\text { Mean } \pm \mathrm{SD}\end{array}$ & $\begin{array}{l}\text { Conservative } \\
\qquad n=43 \\
\text { Mean } \pm \text { SD }\end{array}$ & $\begin{array}{c}\text { Significance } \\
\text { test }\end{array}$ & $\begin{array}{c}\text { Alive } \\
\mathrm{N}=36 \\
\text { Mean } \pm \mathrm{SD}\end{array}$ & $\begin{array}{c}\text { Dead } \\
\mathrm{N}=24 \\
\text { Mean } \pm \mathrm{SD}\end{array}$ & $\begin{array}{c}\text { Significance } \\
\text { test }\end{array}$ \\
\hline Systolic Bl. $\backslash \mathrm{P}$ & $118.3 \pm 18.6$ & $127.1 \pm 25.8$ & $\mathrm{P}=0.2$ & $116.9 \pm 17.5$ & $135.8 \pm 28.1$ & $\mathrm{P}=0.006^{*}$ \\
\hline Diastolic Bl. \P & $73.9 \pm 6.9$ & $78.1 \pm 14.2$ & $\mathrm{P}=0.1$ & $\begin{array}{r}72.8 \\
\pm 10.03 \\
\end{array}$ & $82.9 \pm 13.7$ & $\mathrm{P}=0.002^{\star}$ \\
\hline INR & $1.2 \pm 0.1$ & $1.2405 \pm 0.2$ & $\mathrm{P}=0.9$ & $1.2 \pm 0.2$ & $1.3 \pm 0.2$ & $\mathrm{P}=0.4$ \\
\hline ABGPH & $7.29 \pm 0.1$ & $7.34 \pm 0.07$ & $\mathrm{P}=0.04^{\star}$ & $7.3 \pm 0.1$ & $7.3 \pm 0.07$ & $\mathrm{P}=0.7$ \\
\hline ABG.PCO2 & $37.7 \pm 11.1$ & $35.5 \pm 8.8$ & $\mathrm{P}=0.4$ & $36.4 \pm 9.1$ & $35.7 \pm 10.3$ & $\mathrm{P}=0.8$ \\
\hline ABG.HCO3 & $20.7 \pm 3.2$ & $20.1 \pm 4.2$ & $P=0.6$ & $20.5 \pm 3.8$ & $20.01 \pm 4.2$ & $P=0.7$ \\
\hline ABG: $\mathrm{Na}$ & $147.1 \pm 8.3$ & $148.8 \pm 8.1$ & $\mathrm{P}=0.5$ & $145.9 \pm 7.3$ & $151.9 \pm 8.04$ & $\mathrm{P}=0.004^{*}$ \\
\hline ABG.K & $3.4 \pm 0.5$ & $3.3 \pm 0.5$ & $\mathrm{P}=0.3$ & $3.4 \pm 0.5$ & $3.2 \pm 0.6$ & $\mathrm{P}=0.2$ \\
\hline WBCs & $\begin{array}{c}19370.6 \pm \\
3954.1\end{array}$ & $16786.1 \pm 5172.9$ & $\mathrm{t}=1.9, \mathrm{P}=0.07$ & $\begin{array}{c}17530.6 \pm \\
5464.99612\end{array}$ & $\begin{array}{c}17500 \pm \\
4222.50698\end{array}$ & $\mathrm{t}=0.02, \mathrm{P}=0.9$ \\
\hline $\mathrm{HB}$ & $11.3 \pm 1.6$ & $11.5 \pm 2.2$ & $\mathrm{t}=0.3, \mathrm{P}=0.7$ & $11.1 \pm 2.3$ & $11.9 \pm 1.5$ & $\mathrm{t}=1.7, \mathrm{P}=0.1$ \\
\hline Platelets & $\begin{array}{l}248823.5 \\
\pm 72919.7\end{array}$ & $\begin{array}{c}233253.5 \\
\pm 101687.2\end{array}$ & $t=0.6, P=0.6$ & $\begin{array}{c}249083.3 \pm \\
100879.7\end{array}$ & $\begin{array}{c}220537.5 \pm \\
81934.09\end{array}$ & $\mathrm{t}=1.2, \mathrm{P}=0.3$ \\
\hline RBS & $171.9 \pm 63.9$ & $157.1 \pm 55.1$ & $\mathrm{t}=0.9, \mathrm{P}=0.4$ & $135.7 \pm 34.4$ & $199.8 \pm 64.2$ & $\mathrm{t}=4.5, \mathrm{P} \leq 0.001^{\star}$ \\
\hline Sr. creatinine & $0.9 \pm 0.2$ & $0.8 \pm 0.3$ & $\mathrm{t}=0.5, \mathrm{P}=0.6$ & $0.7 \pm 0.3$ & $1.02 \pm 0.3$ & $\mathrm{t}=3.7, \mathrm{P} \leq 0.001^{\star}$ \\
\hline
\end{tabular}

*There is significant correlation between $\mathrm{PH}$ and management $(\mathrm{P}=0.04), \mathrm{Sr}$. Na and outcome $(\mathrm{P}=0.004)$, ALT and outcome $(\mathrm{P}=0.03)$, Sr. creatinine and outcome $(\mathrm{P} \leq 0.001)$.

\section{Discussion}

We discussed in this study different risk factors which affect management and outcome of TBI patients. These risk factors include age, sex, cause of injury, systemic diseases, drug abuse, GCS on admission, and type of lesion.

In the present study we found that children and adults had better outcome than elderly who had poor outcome. There is significant correlation between age and outcome $(\mathrm{P}=0.001)$, especially child group and outcome $(\mathrm{P}=0.003)$. Some studies found that increasing age is a strong independent factor in prognosis of TBI, with a significant increase in fair outcome in patients older than 60 years of age, 
that's due to a higher rate of co-medication and anticoagulants administration which increase the extent of intracerebral bleeding ${ }^{89}$. Xueyan et al. ${ }^{57}$, illustrated in his study, several factors were found to contribute to the poor outcome in elderly patients, including lower Glasgow Coma Scale scores, existing systemic diseases as hypertension, systemic complications, midline shift, and inefficient intensive care unit.

In the present study, we found that incidence of TBI in male was higher than female with a ratio of (5:1) respectively which indicates that male gender was a risk factor for TBI due to the higher incidence of RTA which affects mainly males who are the main workers and drivers in Egypt although sex did not affect outcome of TBI. This finding was in agreement with Taha's (2015) results of his study which was about closed severe traumatic brain injury and was done in Egypt. He found that out of 80 cases, 71 male (88.8\%), and the rest are female $(11.2 \%)$ with mean age of $27.45 \pm 16.46$ year.

Brock's et al. ${ }^{10}$ also reported that the incidence of TBI in male was $12-16 \%$ and $8 \%$ in females so men were more likely to be admitted in hospital and are nearly three times more likely to die after TBI. Albrecht et al. ${ }^{2}$ found that same mortality following isolated TBI among older adults in both sex.

In the present study, we found that the most frequent causes of TBI were road traffic accident, falling from height, struggle and direct head trauma (DHT) respectively. There is statistically significant correlation between age of patient and the cause of injury ( $\mathrm{P}$ value $=0.001)$. Falls represented the most common cause of traumatic brain injury in extremes of age group while RTA and struggle were the most common causes in adult group.

This finding was in agreement with Taha's (2015) results of his study which was done in Egypt, as he reported that RTA was the most important cause of head injury in 52 (65\%) of patients with severe TBI followed by falls in 12 (15\%) of those patients.

Tagliaferri and his collagues ${ }^{52}$ and Raja et al. ${ }^{47}$ considered road traffic accidents is the commonest causes of head injury in adults (40\%) and falls (37\%). The commonest cause of injury was pedestrian (36\%), and falls (24\%) In children.

Maas et.al. ${ }^{32}$ illustrated in his study that the incidence of TBI was increasing due to the increased use of motor vehicles especially in middle and low income countries but in high income countries which have traffic safety regulations result in decline in traffic-related TBI. Vulnerable road users (pedestrians, cyclists, etc.) were particularly at risk.

According to systemic diseases and substance abuse we found that patients with systemic diseases had poor prognosis and worse outcome than other patients who did not have systemic diseases. There is significant correlation between DM, HTN and outcome $(\mathrm{P}=0.001), \quad(\mathrm{P}=0.002) \quad$ respectively. Lustenberger et. al. ${ }^{31}$ found that TBI associated with diabetes mellitus have an almost 1.5 fold increased mortality when compared to patients with isolated TBI. They reported significantly increased in-hospital mortality in patients with vs without DM ( $22.6 \%$ vs. $16.8 \%$. $\mathrm{p}=0.002$ ). 
Timur et. al. ${ }^{54}$ reported in his result that patients with TBI and pre-hospital HTN showed significantly higher mortality than TBI patients with normotensive blood pressure $(13.5 \%$ vs. $25.3 \%, \mathrm{p}<0.001)$. Changes in blood pressure during the pre-hospital period, also resulted in higher hospital mortality.

Rixen et.al. ${ }^{48}$ illustrated the mechanism by which HTN increase morbidity and mortality through elevating cerebral perfusion pressure, which leads to enforced dilatation of cerebral arterioles and rise in cerebral blood volume with elevating intra-cerebral pressure. This in turn leads to deterioration of the blood brain barrier with inversion of the hydrostatic gradients and finally to the formation of cerebral edema and/or hemorrhage.

In our study, we found that there is significant correlation between ALT and outcome $(P=0.03)$. Lustenberger et.al. ${ }^{30}$, illustrated that TBI with liver disease was associated with two-fold increased mortality.

In our study, we found a significant effect of chronic kidney diseases on outcome ( $\mathrm{p} \leq$ 0.001 ) and this finding was in agreement with Liao et. al. ${ }^{29}$ results as they found a significant difference between TBI with end stage renal disease patients and patients without renal disease on mortality $(\mathrm{P} \leq 0.0001)$. This is due to a greater number of comorbidities, such as diabetes mellitus, stroke, systemic hypertension, and heart disease in those patients than non-ESRD patients admitted to the ICU.

In the present study, we found that only one case of drug abuse which had good outcome, may be other cases of drug abuse were missed, this was due to lack of screening tests for substance abuse to patients with TBI in our hospital. O'Phelan et.al. ${ }^{43}$ unexpectedly found that alcohol intake to be associated with decreased mortality. Mathias and Osborn (2016) found that alcohol had either deleterious or protective effects on the brain after a TBI and pre-injury alcohol abuse only had a very limited impact on the cognitive outcomes of their intoxicated mild TBI sample.

Alternatively, Dinh et. al. (2014) found that alcohol consumption may lead to more serious injuries which, in turn, may contribute to poorer cognitive outcomes. Intoxicated patients affecting GCS through decreasing it by $2-3$ points are challenging to classify and should be treated with higher attention (Harry et.al. 2007).

According to GCS at time of admission as a risk factor that affect outcome we found that cases with GCS 9 had worse outcome than cases with GCS 12. So the lower GCS, the more chance for surgical treatment and the worst outcome. Perel et al. (2008) and Agrawal et al. (2012) considered that GCS after full resuscitation was one of the most important predictor factor of outcome after TBI. Baum J. et.al. (2016) illustrated that every 1-point decline in initial GCS at hospital presentation was associated with a $14 \%$ increased risk for fair outcome. Osler et.al. (2016) considered that GCS was an important predictor of mortality in both TBI and non-TBI patients but GCS is a more stronger predictor of death in TBI patients and patients with lower GCS have poor outcome than others. 
In the present study, most patients who survived had GCS 15 on discharge and good outcome and mostly managed conservatively. We determined outcome of patients at hospital discharge. We did not evaluate long term outcomes because difficulty in follow-up of many patients after discharge. Maas et.al. (2008) suggested that outcome after head injury is better to be assessed at 6 months after injury, as experience showed that about $85 \%$ of recovery happened within this time period, but further improvement could occur later.

According to type of lesion, Baum et. al. (2016) found that TBI outcome is more dependent on the severity of the brain injury and less so on extra cranial injuries. In the present study, we found that most common type of hemorrhage was $\mathrm{SDH}$, its incidence was $33 \%$ of cases of the study which was in agreement with Mackenzie (2000) result who found that acute $\mathrm{SDH}$ was the commonest type of traumatic intracranial hematoma.

In the present study, acute EDH incidence was $22 \%$ of cases of moderate head trauma and was common in adult group 9 cases versus 3 children and 1 elderly. Bullock et. al. (2006) illustrated that $\mathrm{EDH}$ is rare in the elderly group, due to dura adherent to the skull. EDH generally had good outcome $27 \%$ of patients were alive but if associated with intracranial lesions outcome become poor (12\% died).

Grandhi et al. (2014) showed that ASDH compared with EDH had worst outcome due to severity of underlying brain damage associated with $\mathrm{ASDH}$, and the rate of mortality are greater especially in elderly patients group with poor initial GCS, and other systemic injuries
In the present study, $\mathrm{SAH}$ was a common intra-cerebral lesion, its incidence was $13 \%$ of moderate head trauma. It had worst outcome especially if associated with other lesion and mostly treated conservatively if not associated with other intra-cerebral lesions. Borczuk et. al. (2013) found that patients with isolated traumatic SAH were at lower risk of deterioration in comparison with patients having other intracranial injuries. Gaetani et al. (1995) considered that SAH was a negative prognostic factor in traumatic head injuries especially if there was an associated intracerebral hematoma. Quigley et al. (2013) illustrated that management of traumatic $\mathrm{SAH}$ was usually conservative in isolated traumatic SAH without other intracranial lesions.

In the present study, cerebral contusions were a common lesion, its incidence was 30\% of moderate head trauma patients and its management was mainly conservative. Generally CC had poor outcome especially if associated with other intra-cerebral lesions. This was in agreement with Houseman et. al. (2012), Soustiel et. al. (2008) and Maas (2016) results who found cerebral contusions were one of the most common traumatic findings and it presented in more than $50 \%$ of patients of moderate and severe TBI. Saeed et al. (2014) result reported not all patients with CC require surgical intervention, $56 \%$ of patients can manage conservative and the remaining $44 \%$ need surgical evacuation, surgery was done for other intracranial lesions. Chieragato et al. (2005) study showed intracranial lesions which increase the chances of a bad neurological outcome were (cerebral 
contusions, subdural hematoma and subarachnoid hemorrhage).

In the present study, we found that fissure fracture was the most common type of fracture, it had good outcome and was treated conservatively especially if not associated with other lesions. Raja et. al. (2013) had confirmed this findings in his study as the dominant type of skull fracture was linear (fissured) fracture in $50 \%$ of cases followed by depressed fracture in $30 \%$, comminuted fracture in $20 \%$.

In the present study, we found only one case of fracture base of the skull that documented on CT scan of the head. Actually there were 15 cases of TBI presented with periorbital hematoma, CSF rhinorrhea andlor CSF otorrhea, bleeding per nose andlor per ear. This finding was explained in Chawla et.al. (2105) study, as to detect a fracture on a CT scan there must be discontinuity of the skull. A linear fracture that comes in the same plane of a CT slice may not be visualised. Linear fractures in the base of the skull were difficult to be identified by CT scan unless depressed or separated.

In the present study, we found 4 cases of DAI and their CT scan was normal presented by disturbed conscious level only and not associated with other injuries and all were treated conservative and had good outcome. Yuh EL (2013) suggested that up to one third of patients presented with mild traumatic brain injury (GCS 13-15) and a normal CT scan upon presentation will demonstrate structural abnormalities on later MR imaging. Joshue et. al. (2013) unexpectedly found that diffuse axonal injury was rarely fatal but was associated with increased possibilities of a poor functional recovery, prognosis was generally considered poor.

In the present study, we found that brain edema was considered a secondary lesion which mostly occurred associated with other lesions and it was present in $20 \%$ of all moderate TBI patients. Adults were more susceptible to brain edema than children. It was mostly treated conservatively and generally had good outcome. Makarenko et. al. (2016) found that secondary brain injury cause a significant morbidity and mortality partly due to brain edema which reduces intracranial compliance and may cause a dangerous rise in intracranial pressure which leads to reduced cerebral blood flow and cerebral perfusion.

In the present study, the incidence of pneumocephalus was $8.3 \%$ of moderate head trauma, occurring in adult more than in children. It always occurred associated with other lesions, treated conservatively and had good outcome.

In the present study, we found that incidence of moderate traumatic brain injury in children was $26.6 \%$, the most common hemorrhages in this group were contusion (31\%), $\mathrm{EDH}(19 \%), \mathrm{SDH}(6.3 \%)$, and $\mathrm{SAH}$ (6.3\%) respectively. Fissure fracture was common in child group (56\%).

We found that adult group were more prone to injury than any age group due to increased incidence of RTA in our country, common hemorrhages in these group were ASDH (41.7\%), contusion (27.8), EDH (25\%), SAH (13.9\%) respectively. The common fracture in this group was fissure (27.8\%), depressed (5.6\%). 
Bullock et. al. (2006) found that elderly population is more liable to hge due to increased fragility of blood vessel walls and increasing antithrombotic and anticoagulant drugs usage. Also we found that the most common hemorrhages in elderly were SDH (50\%) contusion (37.5\%), SAH (25\%), EDH (12.5\%), and $\mathrm{ICH}(12.5 \%)$ respectively.

In the present study, we found that Serum $\mathrm{Na}$ had significant effect on outcome $(\mathrm{P}=0.004)$. Hypernatremia may be attributed to the use of normal saline (0.9\%) for the first 24 hours, the use of hyperosmolar diuretics (mannitol) and loop diuretics (furosemides) and its allowed to have high serum Na till 160 $-170 \mathrm{mmolll}$ in such patients. This cause hyperosmolarity of blood which withdraws water from brain cells and decreasing brain edema.

Our results was in agreement with Maggiore and Picetti (2009) results, they reported the incidence of hypernatremia was significantly related with increased patients mortality $(P=0.003)$. This is due to the coexistence of precipitating factors such as impaired sensorium, altered thirst, central diabetes insipidus, and increased insensible losses. Also, these patients often receive dehydrating measures to reduce cerebral edema and controlling intracranial pressure.

Bradshaw and Smith (2008) and Xing et.al. (2015) illustrated that electrolyte disorder was a common problem in TBI patients who are at a high risk for the development of hypokalemia, hypernatremia. Hypernatremia occurs in TBI patients less commonly than hyponatremia. Hypernatremia is often an indicator of the severity of the underlying disease. Hypokalemia is a common electrolyte disorder in hospitalized patients, with a prevalence of $21 \%$ and according the severity of post-traumatic hypokalemia, the severity of head injury can be assisted.

\section{Conclusion}

The most common cause of injury is road traffic accidents with Cause of injury had statistically significant effect on management and statistically non-significant effect on outcome. Patient gender had no effect on management and outcome. We found significant effect of age of patients, systemic diseases (such as DM, HTN, chronic kidney diseases, and chronic liver diseases), type of lesions (especially SDH, SAH), and serum electrolytes (especially serum Sodium) on outcome which determined by GCS at discharge, length of hospital stay, and the state of the patient at discharge.

\section{References}

1-Agrawal D, Joshua SP, Gupta D, Sinha S, Satyarthee GD. (2012). Can Glasgow coma score at discharge represent final outcome in severe head injury? J Emerg Trauma Shock 5(3):217-9.

2-Albrecht JS, Mc-Cunn M, Stein DM, Simoni-Wastila L, Smith GS. (2016). Sex differences in mortality following isolated traumatic brain injury among older adults. The Journal of Trauma and Acute Care Surgery. 81(3):486492.

3-Andriessen TM, Horn J, Franschman G, van der Naalt J, Haitsma I, Jacobs B, et al. (2011). Epidemiology, severity classification, and outcome of moderate and severe traumatic brain injury: a prospective multicenter study. J Neurotrauma. 28:2019-31.

4-Andriessen TM, Jacobs B, Vos PE. (2010). Clinical characteristics and pathophysiological mechanisms of focal and diffuse traumatic brain injury. J Cell Mol Med. 14(10):2381-2392. 
5-Baum J, Entezami P, Shah K, Medhkour A. (2016). Predictors of Outcomes in Traumatic Brain Injury. World Neurosurg.

6- Borczuk P, Penn J, Peak D, Chang Y. (2013). Patients with traumatic subarachnoid hemorrhage are at low risk for deterioration or neurosurgical intervention. J Trauma Acute Care Surg 74:1504-1509.

7-Bradshaw K and Smith M. (2008). Disorders of sodium balance after brain injury Critical Care \& Pain | Volume 8 Number 4.

8-Brain Trauma Foundation (BTF), American Association of Neurological Surgeons, Congress of Neurological Surgeons, Joint Section on Neurotrauma and Critical Care. (2007). Guidelines for the management of severe traumatic brain injury. Journal of Neurotrauma. 24 Supplement 1:S1-S95.

9-Brain Trauma Foundation (BTF). (2000). Early indicators of prognosis in severe traumatic brain injury. J Neurotrauma 17:535-627.

10-Brock F.R. Thomas J. Farrer, Mark Primosch, Dawson W. Hedges. (2013). Prevalence of Traumatic Brain Injury in the General Adult Population: A Meta-Analysis. Neuroepidemiology, 40:154-159.

11- Bullock M.R., Chesnut R., Ghajar J., Gordon D., Hartl R., Newell D.W. et al. (2006). Surgical Management of acute epidural haematomas. Neurosurgery vol. 58 :) Supplement) 52-7.

12-Bullock M.R., Chesnut R, Ghajar J, Gordon D, Hartl R, Newell DW, Servadei F, Walters BC, Wilberger JE. (2006). Surgical Management of Acute Subdural Hematomas. Neurosurgery 58:S2-16-S2-24.

13-Bullock MR, Chesnut R, Ghajar J, et al. (2006). Surgical management of depressed cranial fractures. Neurosurgery, 58:S56.

14- Bullock RM, Chesnut R, Ghajar J, Gordon D, Hartl R, Newell DW, et al. (2006). Surgical management of traumatic parenchymal lesions. Neurosurgery. 58:S2-2546.

15-Chavli K. H., Sharma B. R., Harish D., Anup Sharma. (2006) Head injury: The principal killer in road traffic accidents. JIAFM, 28(4) ISSN: 0971-0973.

16-Chawla H., Yadav KR, Griwan SM, Malhotra R, and Paliwal KP. (2015). Sensitivity and specificity of CT scan in revealing skull fracture in medico-legal head injury victims. Australas Med J. 8(7): 235-238.

17-Chieragato A, Fainardi E, Morselli-Labate AM, Antonelli V, Compagnone C, Targa L, Kraus J, Servadei
F. (2005). Factors associated with neurological outcome and lesion progression in traumatic subarachnoid hemorrhage patients. Neurosurgery. 56:671-680.

18-Christopher Wood G. and Bradley A. Boucher. (2011). Management of Acute Traumatic Brain Injury. PSAPVII. Neurology and Psychiatry.

19-Compagnone C., d'Avella D., Servadei F., Angileri F.F., Brambilla G., Conti C., et al. (2009). Patients with moderate head injury: a prospective multicenter study of 315 patients. Neurosurgery, 64:690-697.

20-Daniel Agustın Godoy, Andre's Rubiano, Alejandro A. Rabinstein, Ross Bullock. Juan Sahuquillo. (2016). Moderate Traumatic Brain Injury: The Grey Zone of Neurotrauma. Neurocrit care, page 4.

21-Fabbri A, Servadei F, Marchesini G, Stein SC, Vandelli A. (2008). Early predictors of unfavourable outcome in subjects with moderate head injury in the emergency department. J Neurol Neurosurg Psychiatry, 79:567-73 22-Frattalone AR, Ling GSF. (2013). moderate and severe traumatic brain injury: pathophysiology and management. Neurosurg Clin N Am. 24(3):309-319.

23-Gaetani P, Tancioni F, Tartara F, Carnevale L, Brambilla G, Mille T, et al. (1995). Prognostic value of the amount of posttraumatic subarachnoid haemorrhage in a six month follow up period. J Neurol Neurosurg Psychiatry; 59:635-7.

24-Grandhi R, Bonfield CM, Newman WC, Okonkwo DO. (2014). surgical management of traumatic brain injury: a review of guidelines, pathophysiology, neurophysiology, outcomes, and controversies. J Neurosurg Sci. 58:249-59.

25-Harry McNaughton, Michael Ardagh, Andrew Beattie. (2007). Traumatic Brain Injury: Diagnosis, Acute Management and Rehabilitation ACC2404. New Zealand guidelines group for ACC.p.4.

26-Houseman C, Belverud S, Narayan R. Closed Head Injury. In: Ellenbogen R, Abdulrauf S, editors. (2012). Principels of neurological surgery, Philadelphia: Saunders Elsevier; pp. 325-47.

27- Joshua M. Levine and Monisha A. Kumar. (2013). Traumatic Brain Injury. Neurocritical Care Society Practice Update.P6.

28-Langlios J.A. \& Sattin, R.W. (2005). Traumatic brain injury in United States: Emergency Department Visits, Hospitalizations, and Deaths research and programs of the Centers for Disease Control and Prevention (CDC). J.Head Trauma Rehabil. 20,187-188. 
29-Liao JC, Ho CH, Liang FW, Wang JJ, Lin KC, Chio CC, Kuo JR. (2014). One-Year Mortality Associations in Hemodialysis Patients after Traumatic Brain Injury. An Eight-Year Population-Based Study. PLoS ONE 9(4). 30-Lustenberger T, Talving P, Lam L, Inaba K, Branco BC, Plurad D, Demetriades D. (2011). Liver cirrhosis and traumatic brain injury: a fatal combination based on National Trauma Databank analysis. Am Surg. Mar; 77(3):311-4.

31-Lustenberger T1, Talving P, Lam L, Inaba K, Bass M, Plurad D, Demetriades D. (2013). Effect of diabetes mellitus on outcome in patients with traumatic brain injury: a national trauma databank analysis. Brain Inj. 27(3):281-5.

32-Maas A. (2016). Traumatic brain injury: Changing concepts and approaches, Chinese Journal of Traumatology xxx 1-4.

33-Maas A., Stocchetti N., Bullock R. (2008). Moderate and severe traumatic brain injury in adults. Lancet Neurol. 7: 728-41.

34-Maas Al, Hukkelhoven CW, Marshall LF, Steyerberg EW. (2005). Prediction of outcome in traumatic brain injury with computed tomographic characteristics: a comparison between the computed tomographic classification and combinations of computed tomographic predictors. Neurosurgery J. 57:1173.

35-MacKenzie EJ. (2000). Epidemiology of injuries: current trends and future challenges. Epidemiol Rev. 22:112-119.

36- Maggiore U., Picetti E., Antonucci E., Parenti E., Regolisti G., Mergoni M., Vezzani A., Cabassi A. and Fiaccadori E. (2009). The relation between the incidence of hypernatremia and mortality in patients with severe traumatic brain injury. Critical Care,13:R110.

37-Makarenko S, Griesdale E. D., Gooderham P., Sekhon M.S. (2016). Multimodal neuromonitoring for traumatic brain injury: A shift towards individualized therapy. Journal of Clinical Neuroscience 26, 8-13.

38-Mathias J. L. and Osborn A. J. (2016). Impact of dayof-injury alcohol consumption on outcomes after traumatic brain injury: A meta-analysis. Neuropsychological Rehabilitation.

39-McHugh GS, Engel DC, Butcher I, et al. (2007). Prognostic value of secondary insults in traumatic brain injury: results from the IMPACT study. J Neurotrauma; 24:287.
40- Montaser, Ahmed Hassan, Ahmed Ibrahim. (2013). Epidemiology of moderate and severe traumatic brain injury in Cairo University Hospital in 2010.Scandinavian Journal of Trauma, Resuscitation and Emergency Medicine.21, Suppl 2.

41-National Institute for Health and Clinical Excellence (NICE). (2014). Head injury Triage, assessment, investigation and early management of head injury in children, young people and adults. Clinical guideline Published: 22 January.

42- National Institute of Neurological Disorders and Stroke. (2002). Traumatic Brain Injury: Hope through Research.

43-National Institute of Neurological Disorders and Stroke. NINDS. (2015). Common Data Elements: Traumatic Brain Injury. August 12.

44-O'Phelan, Kristine, McArthur, David L. Chang, Cherylee W. J. Green, Deborah, Hovda, David A. (2008). The Impact of Substance Abuse on Mortality in Patients with Severe Traumatic Brain Injury. Journal of TraumaInjury Infection \& Critical Care: September - Volume 65 - Issue 3 - pp 674-677.

45-Osler T., Cook A., Glance L.G., Lecky F., Bouamra O., Garrett M., Buzas J. S., Hosmer W. D. (2016). The differential mortality of Glasgow Coma Score in patients with and without head injury. Injury, Int. J. Care Injured 47, 1879-1885.

46-Perel P, Arango M, Clayton T, et al. (2008). MRC CRASH Trial Collaborators. Predicting outcome after traumatic brain injury: practical prognostic models based on large cohort of international patients. BMJ. 336(7641):425-429.

47-Quigley MR, Chew BG, Swartz CE, Wilberger JE. (2013). the clinical significance of isolated traumatic subarachnoid hemorrhage. J Trauma Acute Care Surg 74:581-584.

48-Raja R, Verma A, Rathore S. (2013). Pattern of Skull Fractures in Cases of Head Injury by Blunt Force. J Indian Acad Forensic Med. October-December, Vol. 35, No. 4 (0971-0973)

49-Rixen D, Steinhausen E, Dahmen J, Bouillon B, German Society of Trauma Surgery (DGU). (2012). [S3 guideline on treatment of polytrauma/severe injuries. Initial surgical phase: significance--possibilities-difficulties?]. Unfallchirurg. Jan; 115(1):22-9. 
50-Rutland B.W., Langlois JA, Thomas KE, Xi YL. (2006). Incidence of traumatic brain injury in the United States, 2003. J Head Trauma Rehabil. 21:544-548

51-Saeed M., Ahmed S., Ali A. (2014). Current Consideration Regarding Operative Versus NonOperative Outcome of Brain Contusion Patients. Pakistan Journal of Medicine and Dentistry. Vol.3 (01). 52-Soustiel JF, Mahamid E, Goldsher D, Zaaroor M. (2008). Perfusion-CT for early assessment of traumatic cerebral contusions. Neuroradiology.50:189-96.

53- Tagliaferri F, Compagnone C, Korsic M, Servadei, Kraus J. (2006). Asystematic review of brain injury epidemiology in Europe. Acta Neurochir (Wien) 148:255-68.

54-Timmons SD. (2008). Extra-axial hematomas. In: Loftus CM, editor. Neurosurgical Emergencies. 2nd ed: Thieme Medical Publishers. p. 53-67.

55- Timur S., Miersch D., Kienbaum P., Flohé S., Johannes S., Lefering R., and Trauma Registry DGU. (2012). the Impact of Arterial Hypertension on Polytrauma and Traumatic Brain Injury. Dtsch Arztebl Int. Dec; 109(49): 849-856.
56-Tsang KKT, Whitfield PC. (2012). Traumatic brain injury: review of current management strategies. British Journal of Oral and Maxillofacial Surgery 50; 298-308. 57-Xing W., Xin L., Xiangqiong L., Jian Y., Sun Y., Zhuoying D., Xuehai W., Ying M., Liangfu Z., Sirong W., Jin H. (2015). Prevalence of severe hypokalaemia in patients with traumatic brain injury. Injury, Int. J. Care Injured 46, 35-41.

58-Xueyan W., Shengwen L., Wang S., Zhang S., Yang H., Ou Y., Min Z., James L., Shu K., Chen J., Lei T. (2016). Elderly Patients with Severe Traumatic Brain Injury Could Benefit from Surgical Treatment. World Neurosurg. 89:147-152.

59- Yuh EL, Mukherjee P, Lingsma HF, et al. 2013. Magnetic resonance imaging improves 3-month outcome prediction in mild traumatic brain injury. Ann Neurol. 73: 224-235.

60-Zammit C, Knight WA. (2013). Severe traumatic brain injury in adults. Emerg Med Pract.15:1-28. 